\title{
Quasi-static and dynamic behaviour of the bone structures with fine geometric and materials modelling aspects
}

\author{
O. Mayeur, G. Haugou, F. Chaâri, R. Delille, P. Drazetic, and E. Markiewicz \\ LAMIH UMR 8201 - CNRS, University of Valenciennes, 59313 Valenciennes Cedex 9, France
}

\begin{abstract}
The principle aim of this study is to highlight the influence of velocity on mechanical responses of cortical bones. Quasi-static tests are performed on cubic samples from bovine femurs in order to highlight the anisotropic effect of cortical structure. Thanks to the Hopkinson bars technique, a set of curves will be obtained and analysed to define precisely mechanical behaviour of porosity and loading directions. Therefore, this technique combined with a precise geometrical measurement based on $\mu \mathrm{CT}$ technique is expected to provide a more accurate representation of the mechanical behaviour of biological tissues. This protocol will be applied on human tissues after validation of geometrical and material correlation in order to increase the biofidelity of human body models.
\end{abstract}

\section{Introduction}

The decrease of injuries in road accidents is a challenging task and the improvement in this science requires more and more numerical tools for future evaluations of new generation of cars. To access the safety system, numerous models of the human body have already been populated. Labman (Lizee [1]), Thums (Iwamoto [2]), Humos (Behr [3]), were published as incomes of European consortiums or as car manufacturer initiatives. These models are constrained by simplification needed for global testing and are limited in their biofidelity. In this context, the knowledge in biomechanics needs to be increased partly thanks to more accurate studies in mechanical behaviour of the skeleton. This research challenge is based on improvement of the mechanical behaviour of bone. Bone is a hard mineralized tissue consisting of a fibrous, organic matrix bound by inorganic salts (Viano [4]). As a matter of fact, the major difference between cortical bone and cancellous bone is due to the degree of porosity of the material. Cortical bone has porosity under $30 \%$, while cancellous bone has a porosity ranging from 30 to $90 \%$ (Viano [4]). Different mechanical tests on cortical bone are performed during the past decades. This characterisation on biological material is a relevant task due to the anisotropies, heterogeneities and nonlinearities. The strain rates in tensile were studied on bovine bones on quasi-static by Katz [5], and dynamic at strain rate at $100 \mathrm{~s}^{-1}$ (Wright [6], Crowninshield [7] and Ferreira [8]) and on SHTB from $300 \mathrm{~s}^{-1}$ to $1000 \mathrm{~s}^{-1}$ (Parish [9]). The present study tries to highlight the influence of velocity on cortical structure responses through quasistatic and dynamic tests. Thanks to compression testing machines and Hopkinson bars, a set of strain-stress curves is obtained and analysed to define precisely mechanical behaviour of cortical bone on fresh bovine femur. The main interest of this study is to combine the mechanical test to the generation of accurate geometrical measurement.

\section{Material and method}

\subsection{Sample harvesting and preparation}

This study is based on bovine femur as a feasibility step to human bones on future work. Cubic shape sample are harvested from 2 bovine femurs (first one on quasi-static test and second one for dynamic tests). This procedure employed surgical saw for debit step and a precision cut-off machine with diamond wheels (STRUERS, Secotom10) to finalise the sample shape. The first extraction with a surgical saw produces flat coupon of bovine femur. For the quasi-static compression tests, the samples dimensions are about $4 \mathrm{~mm}$. This limited size is adapted to the mechanical test machine. Due to this reduced size of sample, dimensions are not perfect but the measurement of the real section area can be ensured by the micro tomography acquisition of each sample (Fig. 2). The sample location is also determined in order to study its influence on the mechanical behaviour. Concerning the dynamic tests performed on SHPB, the equilibrium forces is controlled by element size. So these tests are carried with $7 \mathrm{~mm}$ cubic samples. This size ensured the best elastic waves' equilibrium. A total of 59 samples has been tested under quasi-static (40) and dynamic (19) configuration (Fig. 1). Samples are tested in axial, radial and transverse direction and porosity is also considered.

Concerning the quasi-static tests, the axial loading configuration is performed on 20 samples divided into 2 categories based on porosity measurement from $\mu \mathrm{CT}$ data. 10 samples without porosity (less than 1\%) are considered in axial loading. This category has a median porosity value at $0.3 \%$ with standard deviation about $0.25 \%$. The second category of axial loading is composed by 10 samples where porosity is higher than $1 \%$. Considering the whole population of this category, the median value of porosity measurement is about $2.18 \%$ for a standard deviation at $1.1 \%$. The 2 other categories concerned the radial (10 samples) and transverse (10 samples) loading directions. These 4 categories are considered in order to highlight the loading direction on the bone structure and porosity effect on the mechanical response. For the dynamic tests, a total of 19 cubic samples from other bovine femur have been used on SHPB and high speed machine. Here the influence of the loading direction also investigated. A set of 8 samples was tested on the axial direction for 3 different velocities. Radial loading configuration was composed of 7 samples extracted from the same bovine femur and tested under 2 velocities. A last dynamic category is focused on 4 samples in transverse loading.

This is an Open Access article distributed under the terms of the Creative Commons Attribution License 2.0, which permits unrestricted use, distribution, and reproduction in any medium, provided the original work is properly cited. 


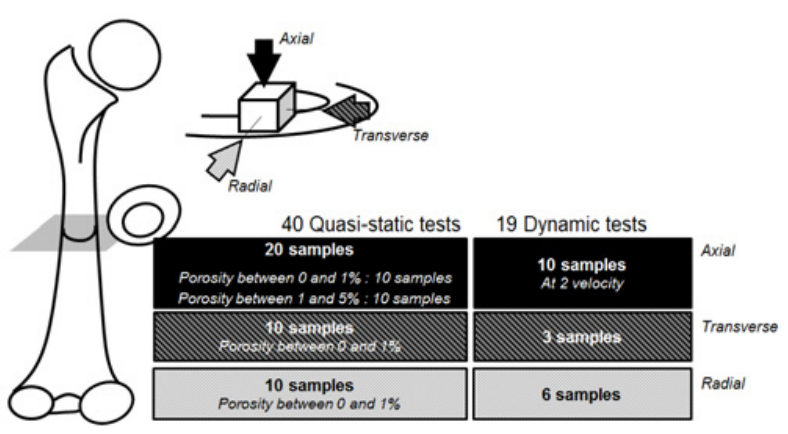

Fig. 1. Description of the loading directions for the test programme on cortical bovine bones.
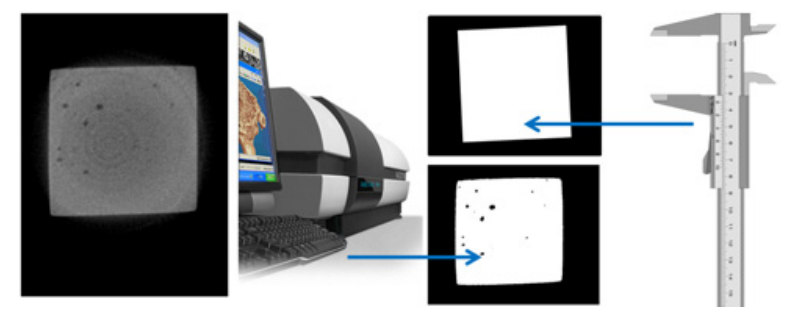

Fig. 2. $\mu \mathrm{CT}$-scan measurement - (a) Bone area cross section of the sample, (b) Comparison between classical cross section measurement and $\mu \mathrm{CT}$ scan area.

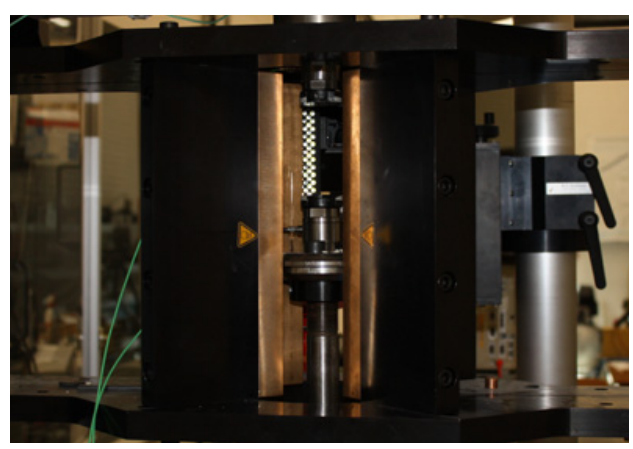

Fig. 3. Compression tests at medium strain rates.

\subsection{Geometrical data acquisition}

Prior to mechanical tests, geometrical measurements are performed on each sample for accurate mechanical behaviour analysis. The apparent density $(\varrho)$ of each specimen was calculated from specimens' weights both in air and water, on the basis of Archimedes' principle. In order to measure precisely the geometry, two kinds of tool are used. Firstly, sample dimensions are taken with an electronic digital calliper: thickness $(\mathrm{h})$, initial length $\left(\mathrm{L}_{0}\right)$ and width (b). Then, the same dimensions parameters are computed from $\mu \mathrm{CT}$ data. Because of the sample size and shape, a $\mu$ CT-scan device was the best application to measure correctly the section bone area $(\mathrm{Ba})$. This measurement gives quantitative information regarding the porosity of sample (fig. 2).

Here, the $\mu \mathrm{CT}$ scanning was performed thanks to an 1172 SKYSKAN system, with $80 \mathrm{kV}$ and $100 \mu \mathrm{A}$ setting. The voxel size was adjusted to $20.8 \mu \mathrm{m}$. A study of shape deviation is also made on the $\mu \mathrm{CT}$ data and controls the different dimensions. The most important observation

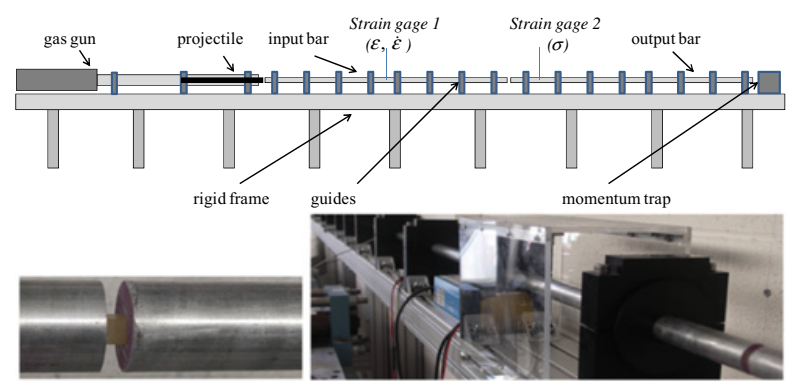

Fig. 4. Set up for dynamic compression tests using aluminium bars.

concerns the section area on useful length that is almost constant $(0.5 \%$ variation $)$.

\subsection{Mechanical testing}

As a large range of strain rates is covered to establish the evolution of cortical bone mechanical behaviour under compression loading, different experimental devices are required. For the lowest range of strain rates, a classical tensile/compression machine is used (Instron ElectroPulse Dynamic E3000) with adapted spherical seated compression platens. The load cell (Dynacell) mounted on the rigid frame has a measuring range up to $3 \mathrm{kN}$. For the medium strain rates, a high speed machine (Instron VHS 65/20) is prepared to performed compression test at $0.05 \mathrm{~s}^{-1}$. For these tests, a piezo electric load cell (MR: $7 \mathrm{kN})$ is threaded at the end of a rod connected to the jack of the machine (Fig. 3). The sample is sandwiched during the test between two platens with reduced mass. The calculation of the shortening of the sample is obtained using an electro optical extensometer (Rudolph XR 200 - MR:10 mm).

A set of compression Hopkinson bars is chosen to catch the range of the higher strain rates. The configuration of the Split-Hopkinson Pressure Bars device is given in a schematic representation (Fig. 4a). The calculation of the material responses is determined on the basis of raw signals collected from full strain bridges bonded at strategic locations along input and output bars so that no superposition of the elastic incident and reflected pulses can occur during the test. Here, the set up is composed of two aluminium bars with a calibrated diameter to $25 \mathrm{~mm}$ and the total length of each bar is equal to $3 \mathrm{~m}$ (Fig. $4 \mathrm{~b}$ ).

On the basis of the overall length of the device, the fullstrain bridge cemented on the input bar is located at midlength of the bar, and the full-strain bridge of the output bar is located at $400 \mathrm{~mm}$ at the interface with the sample. The measurement bars are aligned along a rigid frame made of aluminium so as to confer good parallelism conditions at the location of the sample. The loading of the sample is initiated when the incident pulse $\varepsilon_{\mathrm{INC}}(\mathrm{t})$ - generated during the impact of the projectile with the incident bar - is separated into two complementary elastic pulses called $\varepsilon_{\mathrm{REF}}(\mathrm{t})$ and $\varepsilon_{\text {TRA }}(\mathrm{t})$ (reflected and transmitted pulses) propagating in the input and output bars, respectively. The history of the mentioned elastic pulses is finally converted to strain-stress relations (engineering and true data assuming Poisson' 
ratio correction 0.21 ) using classical governing equations detailed hereafter.

\subsection{Data analysis}

Concerning the quasi-static tests, analyses have performed assuming that cortical structure has an elastic and isotropic behaviour. With respect to these hypotheses, strain-stress curves were obtained with different corrections using the standard normalization relations. The stress correction is made thanks to the section measurement (cortical bone area) instead of the calliper measurement.

As Kolsky technique is here used, current relative displacements and forces are calculated at first on the basis of well-known governing relations (Eqs. (1) and (2).

$$
\begin{gathered}
F_{I N}(t)=S_{I N} \cdot E_{I N}\left(\varepsilon_{I N C}(t)+\varepsilon_{R E F}(t)\right) \\
F_{O U T}(t)=S_{\text {OUT }} \cdot E_{O U T} \cdot \varepsilon_{T R A}(t)
\end{gathered}
$$

Some of them are mentioned below to access to strainstress relations (Eqs. (3) to (6):

$$
\dot{\varepsilon}_{N}(t)=\frac{v_{I N}(t)-v_{O U T}(t)}{l_{S}}
$$

where $v_{I N C}(t)$ and $v_{O U T}(t)$ are respectively the input and output bars' speed and $l_{S}$ is the intial length of the sample. $\varepsilon_{N}(t)$ is the engineering strain finally obtained using a single integration in function of time of relation (3).

$$
v_{I N}(t)=-C_{I N} \cdot\left(\varepsilon_{I N C}(t)-\varepsilon_{R E F}(t)\right)
$$

where $\varepsilon_{I N C}(t)$ and $\varepsilon_{R E F}(t)$ are respectively the amplitude of the incident and reflected pulses propagating in the input bar. $C_{I N}$ is the wave's speed of the incident and reflected pulses propagating in the input bar.

$$
v_{O U T}(t)=-C_{O U T} \cdot \varepsilon_{T R A}(t)
$$

where $\varepsilon_{T R A}(t)$ is the amplitude of the transmitted pulse and $C_{O U T}$ is the wave speed of the transmitted pulse propagating in the output bar.

$$
\sigma_{N}(t)=\frac{S_{O U T} \cdot E_{O U T}}{S_{S}} \cdot \varepsilon_{T R A}(t)
$$

where $S_{\text {OUT }}$ and $S_{S}$ are respectively the cross section of the output bar and the sample. $E_{\text {OUT }}$ is the elastic modulus of the output bar. $\sigma_{N}(t)$ is the current stress calculated in the sample.

Current displacements at intput/output bars and the shortening of the specimen are obtained with $1^{\text {st }}$ order integration in function of time applied on Eq. (4) and (5), respectively. This statement is also admitted for the calculation of the current strain on the basis of Eq. (3). As a very fine geometrical analysis is developed using $\mu \mathrm{CT}$ scanning (see Sect. 2.2), the real cross section of the sample is addressed and gives the opportunity to adjust the calculation of the stress.

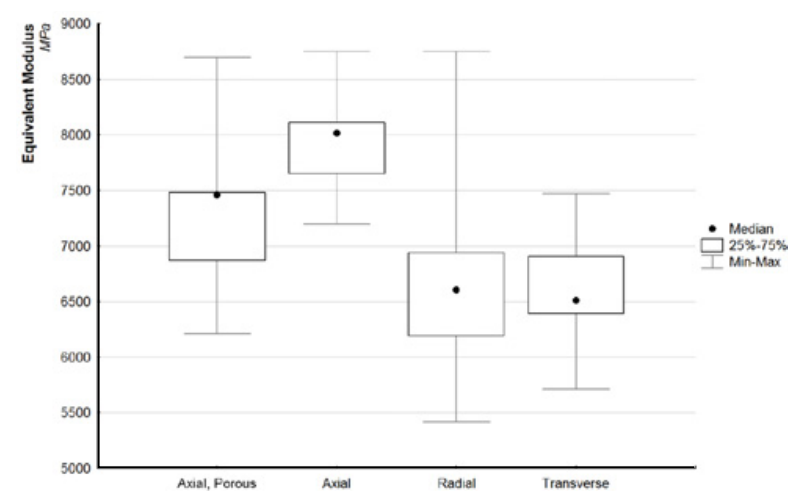

Fig. 5. Equivalent modulus for the 3 loading directions (4 categories).

\section{Results}

The 40 samples used in quasi-static tests are analysed to illustrate the porosity and anisotropic effects on the first bovine femur. Dynamic tests results of the second bovine femur are used to underline the velocity effects and anisotropy of cortical structure.

\subsection{Quasi-static tests}

Quasi-static tests are focused on the mechanical behaviour of the cortical bone regarding the loading directions and porosity effect. A large dispersion is underlined on the 40 samples. In order to relate the differences on mechanical response, the equivalent modulus is plotted in function of the 3 loading configurations (Fig. 5).

The equivalent modulus on the 40 samples is very dispersive with a maximum value at $8750 \mathrm{MPa}$ and minimum at $5500 \mathrm{MPa}$. This scattering is less important when the loading direction is considered. The box-plot representation corresponding to a $50^{\text {th }}$ percentile range is quite similar with standard deviation about $600 \mathrm{MPa}$ for each loading direction classes. The equivalent modulus of the samples tested in axial configuration is higher for the sample where porosity is less than $1 \%$. A decrease of the equivalent modulus is observed when the porosity is considered (median value at $7500 \mathrm{MPa}$ against $8000 \mathrm{MPa}$ without porosity). This result is in agreement with material behaviour. Concerning the radial and transverse loading directions, both median values have an equivalent modulus of $6550 \mathrm{MPa}$ but dispersion is more important on radial tested samples.

In addition, analyses are made on the strain and stress at failure. Strain at failure is similar on axial and transverse loading (4\%) whereas it reaches $7.5 \%$ in radial loading. On the 10 samples where porosity is between $1 \%$ and $5 \%$, the median value of strain at failure is higher by $1.5 \%$ than results in axial direction on sample with less than $1 \%$ porosity. The stress at failure is higher on axial configuration with a median value at $245 \mathrm{MPa}$ for a standard deviation at $22 \mathrm{MPa}$ on samples with less than $1 \%$ porosity. The others axial tests, with porosity, have a median value at $217 \mathrm{MPa}$ with standard deviation at $13 \mathrm{MPa}$. This result is expected since porosity would affects the rigidity. The 


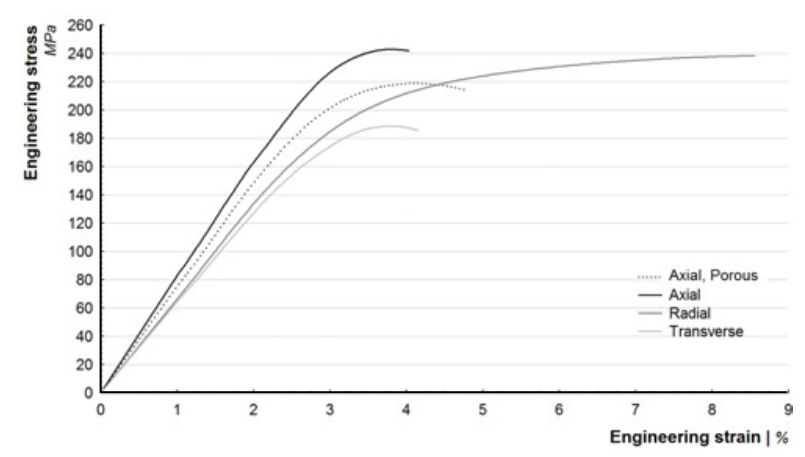

Fig. 6. Mechanical behaviour of cortical bone in compression function of the 4 loading direction configuration.

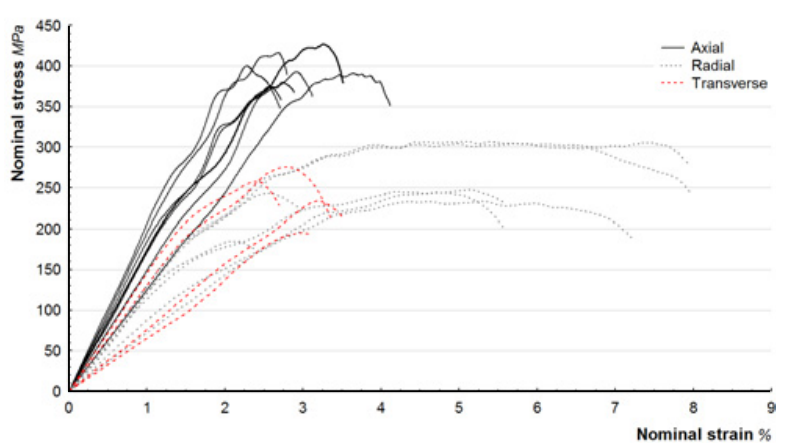

Fig. 7. SHPB results on cortical bone samples in function of the direction of the bone-Compression tests at $500 \mathrm{~s}^{-1}$.

stress at failure is very different in transverse (similar to axial loading with median value about $238 \mathrm{MPa}$ ) and radial direction (median value at $188 \mathrm{MPa}$ ). The different median values are used to plot the different mechanical behaviour of the cortical bone in function of the different loading direction in compression (Fig. 6). Here is revealed the influence of loading direction and porosity parameters on the mechanical response, particularly for the transverse direction where the strain at failure is much higher that all other cases. The influence of porosity on the mechanical response is also put into evidence for loadings in the axial direction of the bone. As the rate of porosity can be considered to correct the strain-stress curve, an increasing of the ultimate stress close to $120 \%$ is observed.

\subsection{Dynamic tests}

The set of 19 samples tested under compression on SplitHopkinson bars gives the oppportunity to check if the observations mentioned below are still available. For higher strain rate, cubic samples are tested in different loading configurations (Fig. 1) on the SHPB at an average strain rate closed to $500 \mathrm{~s}^{-1}$. The different strain-stress curves are plotted on the Fig. 7.

This graph presents the mechanical response in function of the loading directions. Figure 8 gives median values for strain and stress at break coupled with associated standard deviations.

The main observation concerns the radial strain at failure $(5.10 \%)$ which is more important than 2 others
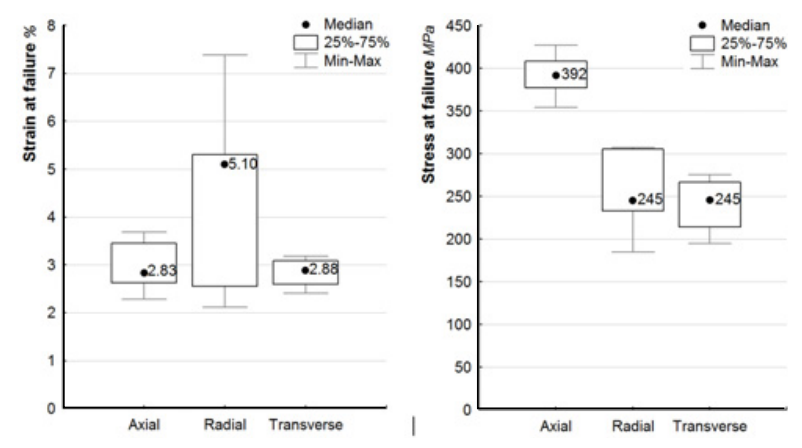

Fig. 8. Mechanical behaviour of cortical bone samples in compression in function of 3 families.

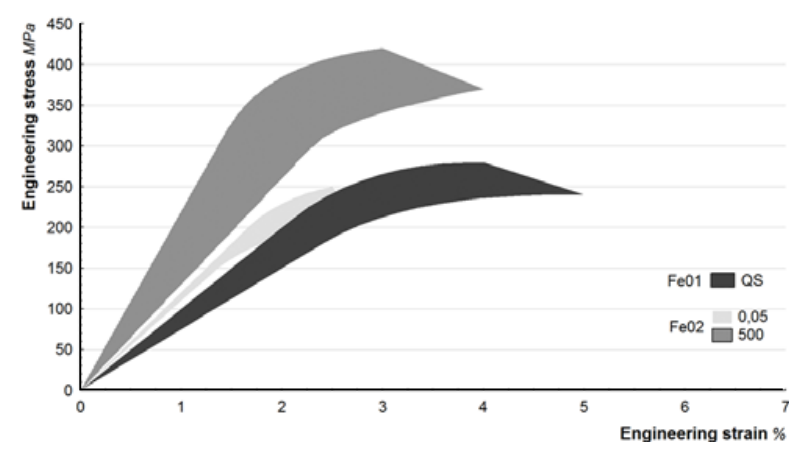

Fig. 9. Mechanical behaviour of cortical bone in the axial direction in function of the strain rate $\left(\mathrm{s}^{-1}\right)$.

directions (axial: $2.83 \%$ and transverse: $2.88 \%$ ). This tendency has been already observed on the quasi-static tests. Concerning the stress at failure, the median value on the axial loading direction is about $392 \mathrm{MPa}$ against $245 \mathrm{MPa}$ in radial and transverse directions. On the same bovine femur, high speed machine has been used for samples loaded in the axial direction at a strain rate about $0.05 \mathrm{~s}^{-1}$. Finally $10^{4}$ decades are covered in terms of strain rates, so that the effect of this parameter on the material responses is clearly put into evidence whatever the direction of loading of the cubic samples. Figure 9 underlines both strain and stress sensitivity regarding the strain rate effect for the compression tests of the fresh bovine samples in the axial direction.

A difference on mechanical behaviour is observed when the strain rate is considered. The compression tests on hydraulic machine (at $0.05 \mathrm{~s}^{-1}$ ) and SHPB $\left(500 \mathrm{~s}^{-1}\right)$ are performed on the same bovine femur ( $\mathrm{fe} 02$ ) and revealed an increase of equivalent modulus and stress at failure as mentioned in literature (Viano [4]). The quasi-static program comes from other bovine femur but confirms the same tendency.

\section{Discussion and conclusion}

In this paper are addressed compression tests on fresh cubic samples extracted from bovine and tests at low, medium and high strain rates (up to $500 \mathrm{~s}^{-1}$ ). The three loading directions of the bone are considered in the identification of the material responses and confirms the 
anisotropy. In parallel, the authors propose a fine description of the imperfections of the cross section of the samples (real shape, porosity) so as to consider geometrical corrections added to the material behaviour of the samples. Biological samples present a natural dispersion which coming from age, conservation, material structure (porosity) and location sample (osteon orientation ... ). In order to reduce this dispersion, an accurate analyse of the tested samples is required and ensured in this study by the $\mu \mathrm{CT}$ scan investigation. The dimension and shape analysis is made prior to testing thanks to $\mu \mathrm{CT}$ data. The location of each sample has been considered and a special attention has been made on the porosity measurement. The Split-Hopkinson pressure bars complete the strain rate effect study. For thus investigation will be done on over vulnerable segment subjected to high speed loading. For instance head, pelvis and ribcage are candidate to tensile and compression test at the scale of coupons. For that, special design of the kolsky technique will be required to extract precisely strain-stress relation.

\section{Acknowledgements}

The present research work is also supported by the International Campus on Safety and Intermodality in Transportation (CISIT), the Nord-Pas-de-Calais Region, the Regional Delegation for Research and Technology, the Department of Higher Education and Research, and the National Centre for Scientfic Research (CNRS). The authors gratefully acknowledge the support of these institutions. This work could not have been done without the generous gift of body donors, through the body donation program of the University of Paris. A special thought to all of them.

\section{References}

1. Lizee E., Robin S., Song E., Proceedings of the $42^{\text {nd }}$ Stapp Car Crash Conference, 115-138 (1998).

2. Iwamoto M., Kisanuki Y., Watanabe I., Furusu K., Miki K., Hasegawa J., Proceedings of International IRCOBI Conference, 31-42 (2002).

3. Behr M., Arnoux P.J., Serre T., Bidal S., Kang H.S., Thollon L., Cavallero C., Kayvantash K., Brunet C., Computer Methods in Biomechanics $\mathcal{F}$ Biomedical Engineering, 6, 263-273 (2003).

4. Viano, D.C., Symposium on Biomechanics and Medical Aspects of Lower Limb Injuries, Technical Paper $\mathrm{n}^{\circ} 861923$ (1986)

5. Katz J.L., Journal of biomechanics, 4, 1-12 (1972)

6. Wright T.M., Hayes W.C., Medical and biological engineering, 14, 671-681 (1976).

7. Crowninshield R.D., Pope M.H., Annals of Biomedical Engineering, 2, 217-225 (1974)

8. Ferreira F., Vaz M., Simoes J., Materials Characterization, 57, 71-79 (2006).

9. Parish A., Chen W., Weerasooriya T., Proceedings of DYMAT Conference, 1, 917-922 (2009). 